\title{
HIGHLIGHT
}

\section{Perinatal programming of appetite control by leptin?}

\author{
Jörg Dötsch, Wolfgang Rascher and Udo Meißner \\ Klinik für Kinder und Jugendliche, Universität Erlangen-Nürnberg, Germany \\ (Correspondence should be addressed to J Dötsch, Department of Pediatrics, Friedrich-Alexander-University Erlangen, Loschgestrasse 15, \\ 91054 Erlangen, Germany; Email: joerg.doetsch@kinder.imed.uni-erlangen.de)
}

The concept of fetal and perinatal programming of neuroendocrine functions has attracted growing interest within the last 15 years. Barker (1) first demonstrated the relationship between low birth weight and diseases of later adulthood such as coronary heart disease and the metabolic syndrome. Meanwhile, there is convincing evidence that intrauterine factors such as placental insufficiency lead to a change of the metabolic and endocrine milieu in the feto-placental interface. These changes influence birth weight and also lead to persistent remodelling of endocrine functions (2, 3). Perinatal programming, therefore, implies persistent epigenetic changes of primarily genetic neuroendocrine functions. Finding the underlying mechanisms for the perinatal programming of a disease such as the metabolic syndrome - a disease associated with tremendous socioeconomic consequences - would be the first step towards initiating innovative concepts for preventive measures.

In a different but related field dealing with the early determination of neuroendocrine function needed later in life, namely the specification of sexually dimorphic patterns in the mammalian forebrain (4), recent advances have been made: specifically the surge of sex steroid secretion in early neonatal life has been linked to neuroanatomical and functional changes in the forebrain $(5-7)$.

With regard to the regulation of appetite and energy consumption, the discovery of the predominantly adipocyte-derived hormone leptin in the mid-1990s has led to a new understanding (8). Leptin, at least in rodents, leads to a decreased food intake by stimulating neurons in the arcuate nucleus of the hypothalamus that contain the anorectic peptide $\alpha$-melanocyte-stimulating hormone $(\alpha-\mathrm{MSH})$ and by inhibiting neurons that coexpress the orexigenic peptides neuropeptide $\mathrm{Y}$ (NPY) and agouti-related peptide (AgRP). These neurons, which in mice are predominantly formed during the second postnatal week, convey information to other parts of the hypothalamus - namely the paraventricular nucleus, the dorsomedial hypothalamic nucleus and the lateral hypothalamic area (9) (Fig. 1).

Due to physiological and pathophysiological leptin resistance in our own species, the concept of appetite regulation can not be completely transferred to humans. More recently discovered trophic functions of leptin appear to be shared by the different species (10). Trophic effects of leptin on the very neurons that transmit its message in the hypothalamus, therefore, could account in part for the - to date missing - links between alterations in energy metabolism and its regulating hormones in fetal and early postnatal life, and the programming of appetite regulation later in life. To prove this hypothesis, several preconditions need to be met:

1. A fetal or early neonatal leptin surge leading to the induction of neuron proliferation. This appears to be the case (5).

2. The effect has to be specific, i.e. leptin must predominantly lead to the formation of hypothalamic neurons that convey the leptin signal and not proliferation in general.

3. In leptin-deficient ob-mice the phenomenon should be missing but should be restored by leptin application to these animals in the critical phase for neuron formation and growth.

4. The trophic effect on neuron formation is only present in the susceptible phase of neuron development.

5. The effect triggered in the developmental phase must persist until adulthood.

In their excellent articles $(9,11)$, Bouret and co-workers address the question whether the neonatal leptin surge in mice is able to induce the formation of nerve fibres that contain $\alpha$-MSH, NPY and AgRP. By implanting crystals of the tracer $1,1^{\prime}$-dioctadecyl-3,3,3',3'-tramethylindocarbocyanine perchlorate (Dil) into the arcuate nucleus of mice the authors could monitor anterograde neuron formation into the surrounding hypothalamic nuclei. They found a ten times lower number of labelled neurons in the arcuate and the paraventricular nucleus when comparing leptin-deficient $o b$-mice to wild-type littermates. This profound reduction was still demonstrated in the adult animals at day 60 of life. The decrease in neuron formation was not found in other hypothalamic nuclei or nerval projections to the limbic system, demonstrating the selectivity of this effect. The fibres that were reduced could indeed be identified to be those containing $\alpha$-MSH and AgRP using immunostaining techniques. This demonstrates that fibres known to transmit the leptin message were involved. 


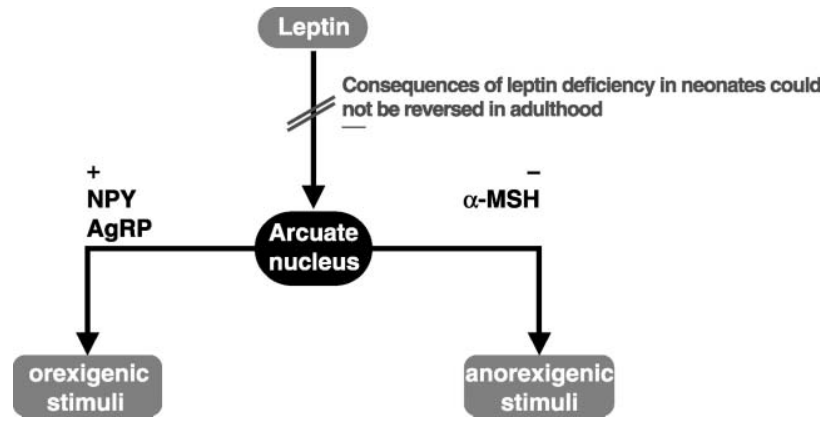

Figure 1 Development of nerve fibres in rat hypothalamus during the first months of life. Impact of leptin on the growth of orexigenic and anorexigenic nerve fibres originating from the hypothalamic arcuate nucleus.

Leptin treatment of neonatal $o b$-mice on days 4-12 of life reversed the reduction in nerve fibres underlining the specific role of leptin in neuron formation to a level seen in wild-type littermates (9). Most importantly, treatment of leptin-deficient adult mice with leptin for 20 days did not result in the formation of anorexigenic and orexigenic nerve fibres. This confirms that there is a critical period for the formation of leptin-dependent neurons in mice.

Interestingly, in the same issue of Science in which the article by Bouret and co-workers (11) appeared, Pinto and colleagues (12) present data on the role of leptin on synapse formation in the arcuate nucleus of the hypothalamus. In adult $o b$-mice leptin leads to the formation of new synapses even if the animals had been leptin deficient before. This suggests that leptin still has effects on hypothalamic neuronal plasticity in the adult animal, despite the reduction in anorexigenic and orexigenic nerve fibres.

In summary, the work by Bouret and co-workers (11) suggests a potential mechanism by which changes in leptin concentration during fetal and neonatal life may alter hypothalamic structures that influence food intake and energy metabolism until adult life. This might be the first step in elucidating mechanisms being important to explain the concept of fetal programming of adult disease.

\section{References}

1 Barker DJP. In utero programming of chronic disease. Clinical Science $199895115-128$.

2 Bertram C, Trowern AR, Copin N, Jackson AA \& Whorwood CB. The maternal diet during pregnancy programs altered expression of the glucocorticoid receptor and type 211 beta-hydroxysteroid dehydrogenase: potential molecular mechanisms underlying the programming of hypertension in utero. Endocrinology $2000 \mathbf{1 4 2}$ 2841-2853.

3 Schoof E, Girstl M, Frobenius W, Kirschbaum M, Dörr HG, Rascher W \& Dötsch J. Reduced placental gene expression of $11 \beta$ hydroxysteroid dehydrogenase type 2 and 15-hydroxyprostaglandin dehydrogenase in patients with preeclampsia. Journal of Clinical Endocrinology and Metabolism $2001 \mathbf{8 6}$ 1313-1317.

4 Dörner G. Problems and terminology of functional teratology. Acta biologica et medica Germanica 197534 1093-1095.

5 Ahima RS, Prabakaran D \& Flier JS. Postnatal leptin surge and regulation of circadian rhythm of leptin by feeding. Implications for energy homeostasis and neuroendocrine function. Journal of Clinical Investigation $1998 \mathbf{1 0 1}$ 1020-1027.

6 Madeira MD \& Lieberman AR. Sexual dimorphism in the mammalian limbic system. Progress in Neurobiology $1995 \quad \mathbf{4 5}$ 275-333.

7 Simerly RB. Wired for reproduction: organization and development of sexually dimorphic circuits in the mammalian forebrain Annual Review of Neuroscience 200225 507-536.

8 Halaas JL, Gajiwala KS, Maffei M, Cohen SL, Chait BT, Rabinowitz D, Lallone RL, Burley SK \& Friedman JM. Weight-reducing effects of the plasma protein encoded by the obese gene. Science 1995 $269543-546$.

9 Bouret SG, Draper SJ \& Simerly RB. Formation of projection pathways from the arcuate nucleus of the hypothalamus to hypothalamic regions implicated in the neural control of feeding behavior in mice. Journal of Neuroscience 200424 2797-2805.

10 Dötsch J, Meissner U \& Rascher W. Leptin-induced weight loss is not solely mediated by anorexia. European Journal of Endocrinology $200314811-12$.

11 Bouret SG, Draper SJ \& Simerly RB. Trophic action of leptin on hypothalamic neurons that regulate feeding. Science $2004 \mathbf{3 0 4}$ $108-115$.

12 Pinto S, Roseberry AG, Liu H, Diano S, Shanabrough M, Cai X, Friedman JM \& Horvath TL. Rapid rewiring of arcuate nucleus feeding circuits by leptin. Science 2004304 110-115.

Received 17 May 2004

Accepted 18 May 2004 